La Revue

des Droits

de l'Homme

\section{La Revue des droits de l'homme}

Revue du Centre de recherches et d'études sur les droits fondamentaux

$4 \mid 2013$

Revue des droits de l'homme $-\mathrm{N}^{\circ} 4$

\title{
Circulez, il y a à voir
}

Ce que les Etats-Unis peuvent apprendre de l'Europe en matière de migrations circulaires et de régularisation des étrangers

All or Nothing? What the United States Can Learn from Europe as it Contemplates. Circular Migration and Legalization for Undocumented Immigrants

\section{Patrick Weil}

Traducteur : Johann Morri

\section{(2) OpenEdition}

\section{Journals}

Édition électronique

URL : http://journals.openedition.org/revdh/336

DOI : $10.4000 /$ revdh.336

ISSN : 2264-119X

Éditeur

Centre de recherches et d'études sur les droits fondamentaux

Référence électronique

Patrick Weil, «Circulez, il y a à voir », La Revue des droits de l'homme [En ligne], 4 | 2013, mis en ligne le 20 décembre 2013, consulté le 09 juillet 2020. URL : http://journals.openedition.org/revdh/336 ; DOI https://doi.org/10.4000/revdh.336

Ce document a été généré automatiquement le 9 juillet 2020

Tous droits réservés 


\section{Circulez, il y a à voir}

Ce que les Etats-Unis peuvent apprendre de l'Europe en matière de migrations circulaires et de régularisation des étrangers

All or Nothing? What the United States Can Learn from Europe as it Contemplates. Circular Migration and Legalization for Undocumented Immigrants

\section{Patrick Weil}

Traduction : Johann Morri

\section{NOTE DE L'ÉDITEUR}

Comme nous voulions que Patrick Weil participe à ce dossier, il a accepté de mettre à notre disposition une version traduite et remaniée d'un de ses articles de référence : «All or Nothing? What the United States Can Learn from Europe as it Contemplates. Circular Migration and Legalization for Undocumented Immigrants » (The German Marshall Fund of the United States, Paper series 2010). La traduction, effectuée par Johann Morri, a été relue par l'auteur.

1 Imaginez un pays que ses nouveaux habitants, depuis leur première installation au XVII ${ }^{\mathrm{e}}$ siècle, ont rejoint par la mer comme s'il s'agissait d'une île. Imaginez encore que le pays dont ces habitants ont hérité et emprunté les lois soit une île. Que dans ce dernier, les frontières aient été contrôlées à l'entrée dans les ports et que la liberté de circulation ait été garantie et vénérée une fois à l'intérieur du territoire. Imaginez ensuite que soudainement, au cours $d u X X^{e}$ siècle, le premier pays soit de moins en moins rejoint par la mer et prenne conscience de ses importantes frontières terrestres.

$\mathrm{Du}$ point de vue de la politique de l'immigration, géographie et politique ne correspondent pas toujours. L'Australie et la Nouvelle-Zélande sont géographiquement et politiquement des îles; le Canada n'est pas un pays insulaire du point de vue géographique, mais politiquement et dans la gestion de sa politique d'immigration, il l'est de facto : les immigrants ne peuvent pas encore facilement atteindre le Canada par 
la frontière terrestre située au Nord. Depuis le Sud, quand ils se trouvent aux EtatsUnis, peu d'immigrants en situation irrégulière sont désireux malgré les difficultés, de quitter un pays où se trouvent plein opportunités et renoncer à un accès aisé au marché du travail.

Du point de vue des flux et de la politique migratoire, les Etats-Unis constituaient une île jusqu'au milieu du $\mathrm{XX}^{\mathrm{e}}$ siècle, dans la mesure où une grande majorité des immigrants et des visiteurs arrivaient par bateau ${ }^{1}$. En outre, les Etats-Unis ont hérité une grande partie de leurs traditions et structures juridiques des habitudes insulaires de la Grande-Bretagne.

Cependant, la géographie politique des Etats-Unis a maintenant changé. Après la mise en œuvre du programme Bracero ${ }^{2}$, l'immigration du Nouveau monde est devenue la principale source de nouveaux migrants ${ }^{3}$. Fondé sur un accord signé le 4 avril 1942 par les Etats-Unis et le Mexique, le programme Bracero a été initialement développé pour résorber la pénurie de main-d'œuvre qui sévissait dans l'agriculture américaine en raison de l'engagement du pays dans la Deuxième guerre mondiale. Toutefois, ce programme s'est poursuivi bien après la fin du conflit, prenant finalement fin en 1964. A son apogée, au milieu des années 50, il apportait plus de 400000 travailleurs mexicains par an aux Etats-Unis ${ }^{4}$. L'augmentation de l'immigration illégale fut un effet collatéral du programme Bracero: «les Braceros qui retournaient au pays faisaient savoir qu'il existait des possibilités d'embauche aux Etats-Unis» et "la préférence donnée (par beaucoup d'employeurs) aux étrangers en situation irrégulière pour l'embauche de Braceros offrait peu d'incitation aux aspirants Braceros à rester au Mexique en attendant une embauche par la voie légale $»^{5}$.

5 En théorie, l'Immigration and Nationality Act de 1965 fut le premier texte à limiter l'émigration de l'hémisphère occidental (120000 personnes). En pratique, les flux migratoires en provenance de cette région - légaux et illégaux - continuèrent à augmenter et à excéder ces chiffres. En 2008, le Mexique était de loin le premier pays de provenance de l'immigration légale aux Etats-Unis, avec 188000 des 1107000 immigrants légaux admis cette année-là ${ }^{6}$. Des centaines de milliers de migrants en situation irrégulière franchissent également la frontière Mexique-Etats-Unis chaque année, contribuant à former la population d'étrangers en situation irrégulière, estimée à 11 millions.

6 Aucun autre pays n'a expérimenté un tournant aussi net dans la géographie politique de sa politique de l'immigration. ${ }^{7}$ Tout spécialement dans l'entre-deux-guerres, la situation insulaire des Etats-Unis facilitait un contrôle du nombre d'immigrants arrivant dans le pays. Le système de quota fut mis en place en $1921^{8}$ et fut à la fois restrictif et efficace, dans la mesure où le gouvernement américain pouvait fixer des limites et que ces limites ne pouvaient jamais être dépassées. Quand la frontière mexicaine est devenue une source accrue d'immigration, l'ancien système est devenu moins efficace. Cependant, la réaction politique n'a pas été d'en changer, mais plutôt de tenter de le rafistoler pour lui rendre son efficacité perdue ${ }^{9}$. Les législateurs se sont abstenus de repenser aussi bien les aspects les plus enracinés du système (les coutumes juridiques et le droit de circuler librement à l'intérieur du territoire des Etats-Unis) que les moins enracinés (le système des quotas) : après l'arrivée massive d'immigrants en situation irrégulière, il devint nécessaire de les régulariser. L'Immigration reform and control act (IRCA) de 1986 est un exemple marquant de régularisation. En échange de cette régularisation, le Congrès imposa des sanctions contre les employeurs et 
encouragea les poursuites contre les mariages de complaisance à travers les dispositions connues sous le nom d'IMFA (Immigration mariage fraud amendments). ${ }^{10} \mathrm{Il}$ limita aussi les pouvoirs de régularisation de l'Exécutif. ${ }^{11}$ Les possibilités de suspendre les mesures d'éloignement en cas de conséquences excessivement dures sur la situation personnelle «furent virtuellement éliminée par le Congrès en $1996 »^{12}$. Depuis cette époque, le droit de détenir les demandeurs d'asile ou les étrangers en attente d'éloignement de façon indéfinie a aussi été étendu. Néanmoins, le système demeure défectueux et il est ressenti comme tel: chaque année, le nombre d'immigrants obtenant le droit de séjourner légalement aux Etats-Unis est égal à celui des migrants qui entrent illégalement; mais $51 \%$ des Américains pensent qu'une majorité des immigrants sont en situation irrégulière et $63 \%$ d'entre eux estiment que le gouvernement fait mal ou très mal son travail de régulation de l'immigration ${ }^{13}$. Car malgré tous les nouveaux développements et l'augmentation de l'immigration en provenance des pays voisins, la culture juridique et le cadre général de la législation américaine sur l'immigration sont demeurés structurellement similaires à ce qu'ils étaient avant la Deuxième guerre mondiale. Aujourd'hui, une régularisation est nécessaire, mais quelles sont les contreparties politiques qui seront imposées pour qu'elle aboutisse?

7 Les propositions les plus couramment évoquées comme des possibilités dans le cadre réforme globale de l'immigration sont notamment contenues dans une proposition de loi adoptée par le Senat et en attente de discussion a la chambres des représentants contient notamment: un important renforcement du contrôle des employeurs, l'élimination ou la restriction de certains types de visas (vraisemblablement les visas dits de "diversité " - ceux qui sont réservés aux pays de faible émigration - et ceux accordés aux membres de famille n'appartenant pas à la famille nucléaire) en contrepartie de l'augmentation des visas pour les immigrés qualifiés ou d'un système de points analogue à celui du Canada ou du Royaume-Uni et surtout la construction de $1200 \mathrm{kms}$ de mur le long de la frontière mexicaine, la systématisation d'un mécanisme 'everify' de contrôle de validité du séjour avant embauche, le suivi et l'enregistrement des visas de touristes pour interpellation plus aisée en cas de dépassement de durée de séjour autorisée. La mise en œuvre de ce dispositif de contrôle conditionne l'attribution d'une carte de séjour permanente et la naturalisation ne pourra être demandée que treize ans après cette attribution ${ }^{14}$

8 En plus de ces propositions couramment évoquées et discutées, certaines propositions inspirées de l'Europe pourraient aussi être intéressantes.

9 Dans le domaine de la politique de l'immigration, il n'y pas de réponses simples et pas d'études scientifiques qui puisse suggérer comme gérer parfaitement les flux d'immigration. Les études comparatives entre pays avec des expériences similaires en matière d'immigration sont souvent une bonne façon d'aborder la question dans un tel contexte.

De la même façon que les Etats-Unis, les quatre pays les plus peuplés de l'Europe continentale de l'Ouest $\mathrm{t}^{15}$ - la France, l'Allemagne et l'Espagne - possèdent des frontières terrestres étendues. Comme les Etats-Unis, ce sont des pays développés situés à proximité immédiate de pays en développement avec un PIB plus faible. De plus, ces pays ont en commun une tendance à une protection accrue des droits des immigrants par les tribunaux ${ }^{16}$. 
11 Dans ce contexte commun, les Etats-Unis et l'Europe continentale sont confrontés à un nouveau défi commun, celui de l'adaptation à un monde globalisé dans lequel un nombre grandissant d'individus ont obtenu ou conquis la possibilité de franchir librement les frontières. Entre 1990 et 2010, le nombre de migrants internationaux a augmenté de $37.5 \%$ (de 155 millions à 213 millions). En gardant ces chiffres à l'esprit, un ensemble de mesures possibles et intéressantes inspirées de l'expérience européenne sont envisageables.

\section{Migrations sédentaires contre migrations circulaires}

12 L'inadaptation des règles relatives aux migrations circulaires est une des nombreuses raisons à l'origine de l'immigration irrégulière, alors que cette forme de migration correspond aux souhaits et aux besoins de certains migrants. Le manque de flexibilité dans l'octroi d'un statut de migrant circulaire oblige beaucoup de migrants, qualifiés et non qualifiés, à demeurer dans le pays d'accueil malgré leur inclination personnelle à faire des allers retours. Les Etats nations du $\mathrm{XX}^{\mathrm{e}}$ siècle étaient inertes et aimaient avoir une population stable, des quotas, et des résidents permanents. L'Etat moderne du XXI siècle doit apprendre à gérer les migrations circulaires et à adapter ses politiques publiques pour donner des droits et un statut aux migrants en mouvement.

La possibilité de circuler et de recirculer de façon permanente devrait être offerte aux travailleurs saisonniers ${ }^{17}$.

De telles mesures bénéficieraient au travailleur, à sa famille, à son pays d'origine et au pays d'accueil dont des secteurs économiques entiers (comme l'agriculture et la construction) dépendent du travail immigré saisonnier. Elles réduiraient le nombre d'étrangers en situation irrégulière. En outre, elle permettrait de s'attaquer à une question qui a divisé ceux qui concourent à élaborer la politique migratoire : les EtatsUnis devraient-ils à nouveau accueillir des travailleurs temporaires ? ${ }^{18}$

15 Tous les travailleurs peu qualifiés ne souhaitent pas s'établir aux Etats-Unis avec leurs familles. Si on leur en laissait le choix, beaucoup retourneraient dans leur pays après avoir travaillé aux Etats-Unis pendant six à dix mois. Avec les salaires gagnés, leur pouvoir d'achat serait bien supérieur dans leur pays qu'aux Etats-Unis.

Grâce à internet, Skype et les différents progrès dans les technologies de la communication, il est aujourd'hui plus facile pour un travailleur saisonnier qu'il y a un demi-siècle de rester en contact avec sa famille au pays ${ }^{19}$. Mais les travailleurs saisonniers ne retourneront dans leur pays d'origine et ne quitteront les Etats-Unis que s'ils ont la garantie qu'ils seront en mesure d'y retourner. Dans le cas contraire, ils ont une forte incitation à rester dans le pays en situation irrégulière. Aussi bien les précédents programmes de travail saisonnier que les programmes actuels ont montré qu'en l'absence de cette garantie, les saisonniers tendent à rester dans le pays d'accueil. Mais le choix n'est pas entre un programme raté d'immigration saisonnière et pas de programme du tout. A la suite d'une initiative franco-allemande ${ }^{20}$, depuis 2007 , la Commission européenne a encouragé les migrations circulaires entre les Etats membres et les pays tiers ${ }^{21}$. L'Italie, l'Espagne et la France ont développé des approches nouvelles dans la gestion du travail saisonnier.

17 En Italie, un quota de travailleurs saisonniers est fixé chaque année. L'année suivante, le travailleur saisonnier qui est retourné dans son pays d'origine à la fin du contrat 
dispose d'une priorité pour revenir l'année suivante. Depuis 2009, il n'y a plus de quota pour les travailleurs permanents, mais un quota spécifique demeure pour les travailleurs saisonniers, fixé à 80000 pour 2010. En Espagne, un programme a été négocié avec le gouvernement marocain pour permettre à un plus grand nombre de Marocains d'entrer et de travailler temporairement. On leur garantit le même niveau de salaire et de protection sociale qu'aux résidents permanents et aux nationaux. En échange de leur retour au Maroc, on leur permet de revenir l'année suivante. Le fait que les autorités espagnoles donnent la priorité à des femmes dont les enfants demeurent dans le pays d'origine contribue à un taux de retour de $95 \%$ chaque année.

La Commission européenne a suggéré que les Etat membres développent des permis de séjour multi-annuels pour les travailleurs saisonniers. En France, la loi du 24 juillet 2006 a créé un titre de séjour pluriannuel de trois ans. Mis en œuvre durant l'été 2008, ce titre de séjour a été proposé à 6500 travailleurs saisonniers. Le gouvernement a récemment souligné son « adaptation au public visé $»^{22}$.

Dans le cas des Etats-Unis, on pourrait imaginer un permis multi annuel de cinq ou dix ans, ou même une carte verte pour saisonniers. Ces permis de séjour pourraient être offerts aux citoyens de tous les pays ou négociés avec certains d'entre eux comme le Mexique. En échange de l'octroi de cartes vertes saisonnières pour des milliers ou des dizaines de milliers de travailleurs mexicains, le Mexique pourrait mieux coopérer avec les Etats-Unis dans le contrôle de ses frontières ${ }^{23}$. Ces travailleurs - d'une certaine façon permanents - devraient avoir le droit d'appartenir à des syndicats et d'être protégés par les conventions collectives de branche ${ }^{24}$.

\section{Permettre la circulation et la recirculation des migrants hautement qualifiés}

Sur la question de l'immigration de travail hautement qualifiée, les Etats-Unis étaient autrefois en avance sur l'Europe. Dans beaucoup de pays européens, comme la France et l'Allemagne, s'exprimait une réticence vis-à-vis de l'immigration qualifiée. Ces conceptions étaient parfois basées sur des tendances racistes ou corporatistes et sur le désir de réserver aux nationaux les situations professionnelles les plus enviables dans la société. Les réserves vis-à-vis de ce type d'immigration étaient aussi fondées sur une vision tiers-mondiste, selon laquelle la justice globale et le développement des nations les moins développées seraient freinés par le fait de recruter et de retenir les migrants hautement qualifiés de ces pays.

21 Cette approche était à la fois incohérente et absurde. Si des Africains, des Asiatiques, des Sud-Américains, ou des étudiants d'autres pays hors de l'Union européenne obtiennent un diplôme d'une université européenne et ne veulent pas retourner dans leur pays, ils ne le feront pas. Si les pays européens leur refusent le statut de résident permanent, ils émigreront aux Etats-Unis, au Canada, en Australie et leurs pays d'origine, et les pays dans lesquels ils ont reçu leur formation, ne bénéficieront pas de leurs capacités professionnelles et intellectuelles. Cette concurrence internationale a encouragé la Grande-Bretagne et l'Allemagne, et dernièrement l'Italie et la France, à ouvrir leurs portes aux travailleurs hautement qualifiés.

22 En dépit de leurs convergences, les Etats-Unis et l'Europe n'ont pas la même approche de la question des migrations hautement qualifiées. Des deux côtés, on limite les droits 
des migrants hautement qualifié qu'on souhaiterait attirer, mais de façon différente. Aux Etats-Unis, une fois que le statut de résident permanent est accordé, le résident étranger hautement qualifié est privé de la possibilité de retourner dans son pays d'origine pendant une période de temps significative : la carte de résident permanent (la « carte verte ») est considérée comme invalide si son titulaire a résidé en dehors du pays pour plus d'an.

Certains pays européens limitent le droit des migrants hautement qualifiés d'obtenir un droit au séjour permanent. La France, par exemple, a créé un nouveau type de carte de séjour de trois ans pour cette catégorie de travailleurs. Mais si le travailleur est un ressortissant d'un pays appartenant à une des régions les plus pauvres du monde ${ }^{25}$ cette carte de séjour ne peut être renouvelée plus d'une fois (pour un séjour total de six ans, donc) et peut seulement être délivrée après approbation des autorités du pays d'origine. Cette politique répond aux fortes réserves émises par certain dirigeants africains auprès de l'Union européenne à propos de politiques qui augmenteraient l'émigration de leur main d'œuvre hautement qualifiée. En comparaison, certains pays déjà considérés comme des grandes puissances, comme la Chine, l'Inde et le Brésil, sont moins préoccupés par le souci de perdre leurs citoyens hautement qualifiés et certains pays (comme les Philippines, par exemple) encouragent l'émigration de leurs travailleurs qualifiés. Pourtant, les ressortissants d'Afrique, d'Asie, d'Amérique du Sud, ou d'autre pays tiers à l'Union européenne titulaires d'un diplôme d'une université européenne sont déjà sur le marché mondial de la main d'œuvre, et toute politique de restriction ou de discrimination à leur égard est inefficace et contre-productive. Les premières évaluations de la politique française en la matière ne sont pas bonnes. Elle a découragé les travailleurs hautement qualifiés des pays les plus pauvres de solliciter la délivrance d'un titre de séjour en France.

Il est pourtant dans l'intérêt des Etats-Unis comme de l'Europe que des pays pauvres, instables et sous-développés puissent à leur tour développer des sociétés civiles démocratiques. Les migrants hautement qualifiés formés en Europe ou aux Etats-Unis peuvent prendre une part importante à ce développement démocratique.

Une fois leur statut de résident permanent obtenu aux Etats-Unis, ils ne devraient pas être empêchés de retourner dans leur pays d'origine - pour quelques mois ou pour quelques années - tout en conservant leur statut.

La Commission européenne encourage les migrations circulaires entre les Etats membres et les pays tiers pour les travailleurs hautement qualifiés. Elle émet le souhait que l'on puisse « donner aux gens la possibilité de s'engager dans une activité (affaires, activité professionnelle, volontariat ou autre) dans leur pays d'origine tout en conservant leur résidence principale dans un des Etats membres. Cela concerne des groupes tels que les hommes et femmes d'affaires travaillant dans l'Union européenne et désireux de démarrer une activité dans leur pays d'origine (ou dans un autre Etat tiers), les médecins, les professeurs, et tous les professionnels désireux de soutenir leur pays d'origine en y conduisant une partie de leur activité professionnelle.

Aux Etats-Unis, trois propositions pourraient être faites dans le but de faciliter la circulation des personnes hautement qualifiées :

-1. Les titulaires du statut de résident permanent devraient pouvoir quitter les Etats-Unis pour une période allant jusqu'à trois ans sans perdre leur statut.

-2. Quand des institutions publiques -hôpitaux, universités, centres de recherche- recrutent des migrants hautement qualifiés dans des pays bénéficiant de l'aide américaine au 
développement, ces travailleurs qualifiés devraient se voir offrir la possibilité de pratiquer leurs activités et de travailler dans leur pays d'origine. Avec l'accord de l'agence américaine pour le développement (USAID), leurs salaires devraient être pris en charge par les institutions qui les ont recrutés pendant la durée de leur séjour dans leur pays d'origine. Cela permettrait aux enseignants ou aux médecins de pays comme Haïti, le Rwanda, le Mali ou le Cambodge recrutés par des universités ou des hôpitaux aux Etats-Unis de mettre en œuvre leur expertise dans leur pays d'origine et de participer à son développement, tout en conservant le droit de retourner aux Etats-Unis pour travailler après une période de temps convenue à l'avance.

- 3. Des visas de longue durée permettant la sortie et la réentrée devraient être offerts aux étudiants titulaires de diplômes de troisième cycle délivrés par les universités américaines. 
organisations patronales et syndicales. Ces quotas inclurent les travailleurs immigrés pour la première fois en 1995. Entre 1995 et 2008, le quota est passé de 25000 à 150000. En parallèle, du milieu des années 1980 à 2009, six campagnes de régularisation (sanatorie, en italien) en eurent lieu : en 1986 (120 000 personnes), en 1990 (220 000 personnes), en 1996 (246000 personnes), en 1999-2000 (250000 personnes), en 2003-2004 (750 000 personnes) et finalement en 2009 (300 000 dossiers déposés). Mais depuis 2008, le gouvernement a renoncé à annoncer tout quota. En contrepartie des régularisations les plus récentes, une nouvelle loi a fait de l'entrée et du séjour irrégulier des infractions pénales, passibles de sanctions financières et d'expulsion immédiate. Néanmoins, des étrangers en situation irrégulière ont continué à être régularisés au titre du reliquat des quotas de 2007 et 2008.

L'Espagne a adopté sa première législation d'ensemble sur l'immigration en 1985, dans le cadre de son accession à l'Union européenne. Très restrictive et axée sur le contrôle des frontières ${ }^{26}$, elle a été suivie d'une régularisation (23000 titres de séjour furent délivrés pour 44000 demandes). Le gouvernement a procédé de façon analogue en 1991. L'institution d'une obligation de visa pour les ressortissants des Etats d'Amérique du Sud et d'Afrique du Nord, afin de préparer l'entrée dans l'espace Schengen, a été accompagnée par une nouvelle régularisation, qui a concerné 116000 étrangers pour 133000 demandeurs. C'est à cette époque que l'immigration est devenue en Espagne un phénomène politique et social. Afin de tenter de réguler le flux d'immigration illégale et répondre aux besoins de main d'œuvre, des quotas furent instaurés en 1993 à un niveau allant de 20000 à 30000 par an, réparti de manière quasiment égale entre travailleurs saisonniers et permanents. Ce système a permis à des milliers de travailleurs jusque-là dissimulés d'obtenir un statut légal chaque année, mais a échoué à réguler les flux migratoires. Après l'adoption, en avril 2000, d'une législation visant à développer une politique d'intégration, une nouvelle régularisation a concerné 164000 étrangers sur un total de 250000 demandeurs. L'opération s'est reproduite après l'adoption de la loi d'août 2002 (184 000 régularisations pour 350000 demandes).

En août 2004, le gouvernement de Jose Luis Zapatero a annoncé la régularisation de tous les étrangers travaillant illégalement, à condition qu'ils justifient d'un contrat de travail pour une durée supérieure à six mois. A la fin de l'année 2005, 576000 des 690679 demandes présentées avaient été acceptées ${ }^{27}$. De plus, entre 20000 et 30000 permis inclus dans le quota sont utilisés pour accorder des régularisations chaque année.

Comme en Italie, le système de quota de l'Espagne a été utilisé pour régulariser des étrangers en situation irrégulière. Les quotas ne furent jamais assez élevés, de telle sorte qu'il y a toujours eu un besoin de nouveaux immigrants, et que le débat sur la nécessité d'une régularisation massive n'a jamais cessé. L'impact politique, social et administratif de ces régularisations massives est souvent dommageable. L'administration est désorganisée, on peut assister à l'arrivée soudaine et massive de nouveaux demandeurs en provenance de pays voisins ou des pays d'origine, et les gouvernements et les parlements sont perçus comme étant à l'origine de politiques de l'immigration mal structurées. De plus, ces régularisations peuvent donner lieu à des réactions de type xénophobe et populiste.

Quelle alternative aux régularisations massives?

L'entrée et le séjour des immigrants en France et en Allemagne n'est pas fondée sur des quotas, mais sur le fait de répondre à des critères, comme ceux qui sont fixés pour le 
regroupement familial, ou de justifier d'une offre d'emploi, ce qui entraine moins d'immigration illégale. De plus, ces deux pays disposent d'un mécanisme permanent de régularisation, qui leur permet d'échapper aux régularisations massives. La France a connu de nombreuses régularisations massives et exceptionnelles : en 1972, 1981, 1991 et 1997. Mais, depuis 1998, un mécanisme permanent (et non exceptionnel) et individuel (et non collectif) de régularisation des étrangers en situation irrégulière a été institué et mis en œuvre. Aujourd'hui, sept catégories d'étrangers en situation irrégulière qui ont tissé des liens forts dans le pays ou qui ont de fortes raisons de demeurer en France peuvent obtenir leur régularisation de la part de l'administration. Il s'agit notamment :

- des étrangers qui démontrent résider habituellement en France depuis plus de dix ans (depuis 2006, cette catégorie a été supprimée: les étrangers en question peuvent encore solliciter leur régularisation, mais elle n'est plus de plein droit et est soumise à une décision discrétionnaire de l'administration);

- les parents d'enfants français ;

- les jeunes âgés de 18 ans qui peuvent démontrer qu'ils sont arrivés en France avant l'âge de dix ans ;

- des étrangers qui peuvent se prévaloir de l'article 8 de la Convention européenne de sauvegarde des droits de l'homme et des libertés fondamentales ${ }^{28}$;

- des étrangers malades qui sont atteint d'une affection dont l'absence de soins entraînerait des conséquences d'une gravité exceptionnelle et qui pour laquelle le traitement n'est pas disponible dans leur pays d'origine ;

- des étrangers dont la régularisation est justifiée par des considérations humanitaires ou exceptionnelles (article 32 de la loi du 24 juillet 2006);

- des étrangers sollicitant une régularisation par le travail (article 12 de la loi du 20 novembre 2007).

Chaque année, 25000 à 30000 délivrances de titres de séjour ( $20 \%$ du total des titres délivrés) correspondent à des régularisations.

En Allemagne, la loi du 31 juillet 2004 (section 25) permet la délivrance de titres de séjour "pour raisons humanitaires". Ce dispositif est destiné à s'appliquer, en particulier, aux personnes protégées par la Convention européenne des droits de l'homme. En 2009, 169782 personnes ont bénéficié de ce titre de séjour. Cette loi complète un dispositif existant en droit allemand (section 60 de la loi) qui permet à environ 100000 personnes de bénéficier d'une autorisation de séjour dite "de tolérance » (Duldung) ${ }^{29}$, dans la mesure où l'Etat allemand ne peut pas les expulser. Pourquoi ces lois ont-elles été adoptées ?

- En régularisant les étrangers qui ont montré la plus volonté d'intégration dans la société d'accueil, elles permettent également d'échapper au cycle des régularisations massives avec le coût politique et les tensions sociales qu'elles impliquent.

- Le processus de régularisation individuelle est géré de façon souple en coopération avec l'administration, les syndicats, les associations, qui jouent un rôle de filtre. Il n'empêche pas, cependant, de faire respecter les lois sur l'immigration. Avant qu'un étranger puisse solliciter sa régularisation, il existe un délai dans lequel l'étranger en situation irrégulière peut faire l'objet d'une mesure d'éloignement.

- Il rend le système plus responsable. Une bonne comparaison est celle que l'on peut faire entre le droit de l'immigration et le droit fiscal. S'il n'y avait pas de sanction pour le nonpaiement des impôts, personne ne les paierait. Mais si les inspecteurs des impôts pouvaient 
enquêter sur vos revenus et vos comptes bancaires plus de vingt ans après les faits, on serait dans un régime totalitaire. C'est la même chose avec la politique de l'immigration. Ne pas avoir de sanctions pour l'immigration illégale ouvrirait la voie à une immigration irrégulière massive, mais l'absence de toute perspective de régularisation après avoir vécu pendant dix ans dans un pays aboutirait à une accumulation de ressentiment et créerait une solidarité collective vis-à-vis de la population immigrée en situation irrégulière. First Quota act du 19 mai 1921. Avant 1921, un immigrant qui ne s'était pas fait enregistrer lors de son entrée aux Etats-Unis et souhaitait pouvoir accéder à la naturalisation avait à sa disposition une procédure simple: il lui suffisait d'aller au Mexique ou au Canada et d'entrer à nouveau aux Etats-Unis régulièrement. Le First Quota Act de 1921 a interdit l'entrée aux étrangers dont le pays avait déjà atteint son quota annuel. La difficulté était que, pour un grand nombre d'étrangers étaient entrés aux Etats-Unis avant le 3 juin 1921, aucune décision d'admission n'avait été enregistrée dans les fichiers de l'administration. Le Registry act du 2 mars 1929 a permis de sortir de cette impasse. La loi autorisait la création d'un statut de résident permanent pour tout étranger non éligible à une naturalisation et pour lequel une décision d'admission n'avait pas été enregistrée, mais qui pouvaient démontrer être entrés aux Etats-Unis antérieurement au 3 juin 1921 et y avoir résidé de façon continue depuis lors, et ne pas être sujets à expulsion.

Aujourd'hui, la section de 249 de l'INA (la loi américaine sur l'immigration) dispose qu'un statut de résident permanent peut être attribué à l'étranger qui est entré aux Etats-Unis avant le $1^{\text {er }}$ janvier 1972 et qui a résidé continuellement aux Etats-Unis depuis cette entrée. Mais la plupart des étrangers en situation irrégulière ne peuvent malheureusement pas remplir cette condition.

Pour compléter leur système de législation sur l'immigration, les Etats-Unis pourraient opérer des emprunts au système franco-allemand et au système hispano-italien. Comme en France et aux Etats-Unis, des mécanismes de régularisation individuelle pourraient être mis en place. Comme en Italie et en Espagne, les permis délivrés sur cette base pourraient être décomptés du nombre de permis fixé par les différents quotas. Une Commission indépendante sur l'immigration, si elle était créée, pourrait se voir confier la tâche d'évaluer l'impact de cette régularisation et d'en déterminer les critères.

\section{Conclusion}

Pour l'Etat-nation, l'immigration est un des défis du futur et non un des problèmes du passé. Nous ne croyons pas au maintien de l'Etat sous sa forme actuelle ou à sa disparition, mais plutôt à sa reconfiguration. Parce que les politiques d'immigration vont devenir de plus en plus complexes et variées, elle vont nécessiter des investissements qui n'ont pas été faits dans le passé en vue de coordonner la multiplicité des acteurs publics et privés, de prendre en compte les aspirations de la population migrante, et d'apprendre à ne pas seulement contrôler le flux des migrations mais à organiser la "recirculation » des migrants sur la longue durée. Cela demandera des innovations radicales dans la politique de l'immigration. Faciliter la circulation et la « recirculation» des citoyens à l'étranger ou des étrangers dans nos pays, accorder à chaque catégorie de migrants un régime adapté - pour les saisonniers

La Revue des droits de l'homme, 4 | 2013 
comme pour les migrants hautement qualifiés - sera une des nouvelles tâches de la politique d'immigration du XXI ${ }^{e}$ siècle.

\section{NOTES}

1. Le principal port d'entrée était Ellis Island. Pour les autres, voir Forgotten doors, the Others ports of entry to the United States, M. Mark Stolarik, Ed. Philadelphia, 1988.

2. NDT : terme espagnol signifiant journalier agricole, passé dans le langage courant aux USA pour désigner un saisonnier agricole originaire du Mexique.

3. L. Aleinikoff, 65.

4. Cf. Kitty Calavita, Inside the State: The Bracero Program, Immigration and the INS, Routledge, London, New-York, 1992.

5. Calavita, idem, 32.

6. 2008 Yearbook of immigration statistics, DHS, Office of immigration statistics, 10.

7. Les seuls autres exemples sont ceux de l'Allemagne et de l'Autriche quand le rideau de fer est tombé. Mais le rideau de fer a duré 40 ans et la tradition juridique de l'Autriche et de l'Allemagne s'était construite pendant des siècles, ce qui fait que le changement ne fut pas aussi drastique qu'aux Etats-Unis.

8. Cf. Son-Thierry Ly et Patrick Weil, "The anti-racist origin of the American quota system » Social research, Volume 77, Number 1 (Spring 2010).

9. Cela pouvait être une illustration de la dépendance vis-à-vis des trajectoires historiques, qui explique souvent la conduite des politiques publiques. Cf. Paul Pierson, « Increasing return, path dependence and the study of politics ", The American political Science Review, vol. 94, $\mathrm{n}^{\circ}$ June 2000, pp. 251-267.

10. 1,8 million d'immigrants présentèrent une demande pour le programme ouvert à ceux qui résidaient aux USA depuis 1982 et 1,3 millions au titre du programme réservé aux travailleurs agricoles (Special Agriculture Workers Program -SAW). Cf. Aristide Zolberg, A Nation by design. Immigration policy in the fashioning of America, Harvard University Press, 2006, p. 371.

11. Adam B. Cox et Cristina M. Rodriguez, "The President and Immigration law ", Yale Law Journal, 2009, $119: 101$.

12. Mae N. Ngai, « We need a deportation deadline », Washington Post, June 14, 2005, p. A2.

13. Cf. Transatlantic trends: Immigration, 2009, question 26. Seulement $49 \%$ des habitants des principaux pays de l'Union européenne ont la même opinion de leur gouvernement.

14. S. 744: Border Security, Economic Opportunity, and Immigration Modernization Act 113th Congress, 2013-2015.

15. En 2007, 8.8 \% (7.2 millions) des habitants de l'Allemagne étaient de nationalité étrangère. En 2004, 8.1 \% de la population totale de la France était immigrée (4.9 million). L'Italie a désormais une population de 4 à 5 millions d'immigrants - environ $7 \%$ de la population. Depuis 200, l'Espagne a absorbé plus de 3 millions d'immigrants, ce qui a fait augmenter sa population de près de $10 \%$. La population d'immigrants dépasse désormais 4,5 millions.

16. Cf. Peter Schuck, « The transformation of immigration law », 84 Col. L. Rev. 1-90 (1984).

17. Les travailleurs saisonniers sont nécessaires pour des emplois saisonniers qui sont par nature temporaires tandis que les travailleurs temporaires sont embauchés pour un contrat à durée déterminée dans lequel la nature temporaire vient de la nature du contrat et non de celle du 
travail. Cf. Booth A.L. J.J. et Frank. J., 2002, « Symposium on temporary work, Introduction, The Economic Journal, vol. 112, 182.

18. Cf. Castaneda et Jacoby, note de bas de page 9.

19. Cf. Diminescu Dana, "Le migrant connecté, pour un manifeste épistémologique ", in Migrations société, vol. XVIII, n 102, nov-dec 2005, pp. 275-293.

20. Sur l'initiative de N. Sarkozy et W. Schauble, 26 octobre 2006 cf. Stefan Angenend, Circular migration. A sustainable concept for migration policy?

21. Communication de la commission européenne, $\operatorname{COM}(2007) 248$ final, On circular migration and mobility partnerships between the European Union and third countries, 16 mai 2007

22. Sénat, commission des Finances, question parlementaire 36 de M. Pierre Bernard Reymond sur le budget 2010

23. Une coopération similaire existe entre les pays faisant partie de l'Espace Schengen et les pays frontaliers, comme la Roumanie et la Bulgarie.

24. Au bout d'un certain temps, par exemple une période de dix ans ou cinq ans, ils devraient également se voir offrir un changement de statut.

25. La «zone de priorité solidaire" créée en 1998 inclut une majorité de pays africains, le Vietnam, le Laos, le Cambodge, Haïti, Cuba, la République dominicaine, etc.

26. Les informations qui suivent sont basées sur Francisco Javier Moreno Fuentes, The evolution of migration policies in Spain between external constraints and domestic demands for unskilled labour, CEACS working paper $n^{\circ} 211$ (Madrid Instituto Juan, March 2004), p. 37.

27. Joaquin Arango, Université Complutense de Madrid, audition devant la commission immigration du Conseil de l'Europe, Paris, 11 décembre 2006.

28. L'article 8 de la Convention affirme le droit de mener une vie privée et familiale normale. L'éloignement d'un étranger en situation irrégulière peut constituer une violation de la Convention s'il sépare une famille ou un couple.

29. Dans la dernière loi, ce document est devenu le «certificat de suspension temporaire d'une mesure d'expulsion ».

\section{RÉSUMÉS}

Même si, en matière d'immigration, les Etats-Unis se sont longtemps pensés comme une île, les défis auxquels ils sont aujourd'hui confrontés sont de même nature que ceux des pays d'Europe continentale : l'adaptation à un monde globalisé dans lequel un nombre grandissant d'individus ont la possibilité de franchir librement les frontières. Dans ce contexte, les Etats-Unis gagneraient à s'inspirer de l'expérience européenne dans différents domaines. Les différentes expériences européennes pour développer les migrations circulaires, par opposition aux migrations sédentaires, pourraient être adaptées aux Etats-Unis, notamment pour les travailleurs saisonniers. La circulation des migrants hautement qualifiés pourrait être facilitée, au bénéfice des pays d'origine et $\mathrm{du}$ pays d'accueil. L'instauration d'un système de régularisation permanente, comme il y existe en France, empêcherait l'accumulation d'une population d'étrangers en situation irrégulière, intenable à long terme, et constituerait une alternative aux régularisations massives.

In terms of immigration policy, the United States have thought themselves as an island for a long time, but the challenges to which they are confronted nowadays are very similar to those of the 
countries of continental Europe : adaptation to a globalized world where a growing number of people can cross the borders easily. In this context, the USA could benefit from the European experience in these matters. The different attempts to develop circular migrations, as opposed to sedentary ones, could be adapted in the USA, especially for seasonal workers. The circulation and re-circulation of highly qualified migrants could be made easier, and it would benefit both the USA and the countries of emigration. The creation of permanent individual legalization schemes would avoid the accumulation of a massive population of undocumented immigrants unsustainable in the long term- and would constitute an alternative to wide scale legalization laws.

United States, immigration law, immigration policy, comparative approach, comparison USA/ Europe, Bracero program, circular migrations, sedentary migrations, highly qualified migrations, legalization, legalization programs in France, Spain and Italy, wide scale legalization, amnesty

\section{INDEX}

Mots-clés : Etats-Unis, droit de l'immigration-politique de l'immigration, approche comparative, comparaison Etats-Unis/Europe, programme Bracero, migrations circulaires, migrations sédentaires, migration hautement qualifiée, régularisation permanente, dispositifs de régularisation en France, en Italie et en Espagne, régularisations massives

\section{AUTEURS}

\section{PATRICK WEIL}

Patrick Weil est directeur de recherches au CNRS à l'Université de Paris 1 Panthéon-Sorbonne, Visiting Professor, Yale Law School. Très connu en France pour ses travaux sur les politiques d'immigration, les discriminations et l'histoire de la nationalité et sa participation à des comités de réflexion sur la réforme de diverses législations, ses travaux en langue anglaise sont moins connus dans l'Hexagone. Pourtant, depuis plusieurs années il intervient en qualité de Visiting Professor au sein de la prestigieuse Yale Law School. Après la traduction en langue anglaise de Qu'est-ce qu'un Français? (How to be French? Nationality in the Making since 1789, Duke University Press, 2008) et la publication de plusieurs articles dans des revues américaine, il a publié en 2012 une recherche originale sur l'histoire des dénaturalisations aux Etats-Unis sous le titre The Sovereign Citizen : Denaturalization and the Origins of the American Republic (Penn Press, Nov. 1, 2012). 